\title{
Differences in Yes-associated protein and mRNA levels in regenerating liver and hepatocellular carcinoma
}

\author{
CHENG WANG $^{1}$, LIN ZHANG ${ }^{1}$, QIAN HE $^{1}$, XIAOBIN FENG ${ }^{1}$, JIN ZHU $^{1}$, ZHAOLONG XU $^{1}$, \\ XIAOJUN WANG ${ }^{1}$, FEI CHEN ${ }^{1}$, XIAOWU LI ${ }^{1}$ and JIAHONG DONG ${ }^{1,2}$ \\ ${ }^{1}$ Institute of Hepatobiliary Surgery, Southwest Hospital, Third Military Medical University, Chongqing 400038; \\ ${ }^{2}$ Institute of Hepatobiliary Surgery, General Hospital of PLA, Beijing 100853, P.R. China
}

Received August 1, 2011; Accepted October 14, 2011

DOI: $10.3892 / \mathrm{mmr} .2011 .640$

\begin{abstract}
Yes-associated protein (YAP) has been identified as an oncoprotein that regulates organ size by modulating proliferative and apoptotic activities. The aim of this study was to measure YAP gene expression and protein levels during rat liver regeneration and in liver cancer, and to explore possible correlations between YAP levels and cancer cell proliferation following partial hepatectomy. We examined YAP expression in rat regenerating liver using real-time PCR, Western blotting and immunohistochemical methods. YAP mRNA and protein levels were measured in human hepatocellular carcinoma. The results demonstrated that YAP protein levels were markedly increased in the regenerating liver (2.5-3 times), while YAP mRNA levels decreased slightly $(1.47-2.17$ times $)(\mathrm{P}=0.017)$. YAP mRNA and protein levels were both higher in hepatocellular carcinoma than in para-cancerous tissue. In conclusion, liver regeneration alone elevated YAP protein levels without elevating YAP mRNA levels. YAP mRNA and protein levels were both elevated in liver cancer cells.
\end{abstract}

\section{Introduction}

In recent years, researchers have demonstrated that Hippo tumor-suppressor signaling is a significant factor in controlling organ size in mammals (1-3). Conditional overexpression of Yes-associated protein (YAP), the nuclear effector of this pathway, increases liver size in mice and leads to organomegaly followed by tumor formation (1). Notably, it was shown

Correspondence to: Dr Jiahong Dong or Dr Xiaowu Li, Institute of Hepatobiliary Surgery, Southwest Hospital, Third Military Medical University, Chongqing 400038, P.R. China

E-mail: dongjh301@163.com

E-mail: lixw1966@yahoo.com.cn

Abbreviations: YAP, Yes-associated protein; HCC, hepatocellular carcinoma; $\mathrm{PH}$, partial hepatectomy; TGF- $\beta 1$, transforming growth factor $\beta 1$; IHC, immunohistochemistry; PCT, para-cancerous tissue; $\mathrm{SD}$, standard deviation

Key words: Yes-associated protein, liver regeneration, hepatocellular carcinoma, partial hepatectomy that YAP-induced growth could be reversed by silencing YAP overexpression. However, the potential role of YAP as the size checkpoint in liver regeneration is poorly understood.

YAP was found to be expressed in $62.1 \%(110 / 177)$ of hepatocellular carcinoma (HCC) cases, but only $9.0 \%(16 / 177)$ of non-tumor specimens, suggesting its use as an independent prognostic marker for overall survival in HCC patients (2). YAP was shown to reduce contact inhibition and induce proliferation of cancer cells (4). YAP inactivation may restrict the proliferation of hepatic oval cells and prevent the development of oval cell-derived tumors (5). The YAP gene has been implicated as an oncogene in HCC (6).

Despite the absence of viable HCC tissue on post-treatment CT images, residual microscopic nests of tumors may lead to local recurrence in certain patients (7). Tumor growth in the remaining liver may be accelerated following resection (8), although the mechanism for this remains unexplained. The effect of YAP on residual tumor growth has not yet been studied.

Therefore, the present study investigated liver regeneration and tumor proliferation in relation to YAP levels in YAP-positive HCC cases that had undergone partial hepatectomy.

\section{Materials and methods}

Animals and partial hepatectomy $(P H)$. Male SpragueDawley rats (160-180 g, 6-8 weeks old), purchased from the Laboratory Animal Center of Daping Hospital (Chongqing, China), were maintained at $25^{\circ} \mathrm{C}$ under a $12 \mathrm{~h} / 12 \mathrm{~h}$ light/dark cycle with standard rat chow and water available ad libitum. The experimental procedures of the study were approved by the Institutional Animal Ethics Committee and adhered to the local guidelines for the use and care of laboratory animals. Twelve hours prior to the experiment the rats were fasted but given free access to water.

Seventy percent partial hepatectomies were performed under ether anesthesia via abdominal median incisions (9). Each rat was maintained in an individual cage. Twenty-five rats were randomly assigned to five groups: the 4, 48, 104, 115 and $240 \mathrm{~h}$ groups; with five rats per group. Five specimens from the $0 \mathrm{~h}$ (control) group and the other groups were obtained by excisional biopsy. Following liver resection, 
Table I. Primers used in the study.

\begin{tabular}{lllc}
\hline Gene & \multicolumn{1}{c}{ Forward primer } & \multicolumn{1}{c}{ Reverse primer } & Product length \\
\hline YAP (rat) & 5'-AAGGCTTGACCCTCGTTT-3' & 5'-CTGCTGCTGCTGGTTTGA-3' & $133 \mathrm{bp}$ \\
$\beta$-actin (rat) & 5'-ATATCGCTGCGCTCGTCGTC-3' & 5'-TCTTGCTCTGGGCCTCGTC-3' & $174 \mathrm{bp}$ \\
TGF- $\beta$ 1 (rat) & 5'-CTAATGGTGGACCGCAACAACG-3' & 5'-TCTGGCACTGCTTCCCGAATG-3' & $104 \mathrm{bp}$ \\
YAP (human) & 5'-ACCCACAGCTCAGCATCTTCG-3' & 5'-TGGCTTGTTCCCATCCATCAG-3' $^{2}$ & $257 \mathrm{bp}$ \\
$\beta$-actin (human) & 5'-GGCACTCTTCCAGCCTTCCTTC-3' & 5'-AGGTCTTTGCGGATGTCCACG-3' & $102 \mathrm{bp}$ \\
\hline
\end{tabular}

a fraction of regenerating liver tissue was analyzed at the various time points. At least three animals were used for each experimental time point. The liver tissues $\left(0.5 \times 0.5 \times 0.5 \mathrm{~cm}^{3}\right)$ were either snap-frozen in liquid nitrogen or fixed at $4^{\circ} \mathrm{C}$ in $40 \mathrm{~g} / \mathrm{l}$ paraformaldehyde ( $\mathrm{pH} 7.3$ ) overnight for future study. At the conclusion of the experiments, rats were sacrificed by exsanguination.

Cell lines. NIH/3T3 (CRL-1658, mouse embryo fibroblast) cells were purchased from ATCC (Rockville, MD, USA).

HCC tissues. Ten pairs of HCC and para-cancerous tissue (PCT) samples were acquired from Southwest Hospital (Chongqing) in 2010. All patients were diagnosed with pathologically proven primary HCC. Prior to the surgery, none of the patients had received radiotherapy or chemotherapy. A portion of the specimens was snap-frozen in liquid nitrogen, and a portion was fixed in buffered paraformaldehyde ( $\mathrm{pH}$ 7.3). PCT was dissected with a margin of at least $1 \mathrm{~cm}$ from the tumor edge. All tissues came from patients who had undergone hepatectomies. Each specimen was analyzed in a blinded fashion by Qingliang Wang, a pathology expert.

Written informed consent was obtained prior to collection of these tissues. All clinical trials in this study were approved by an institutional review board prior to patient enrollment.

Immunohistochemistry (IHC). For IHC, paraffin-embedded tissue blocks were cut into $4-\mu \mathrm{m}$ sections. Antigen retrieval was achieved by microwave exposure at $100^{\circ} \mathrm{C}$ in citrate buffer ( $\mathrm{pH}$ 6.0; ZLI-9064; ZhongShan Goldenbridge Biotechnology Co, Beijing, China) for $10 \mathrm{~min}$. After blocking endogenous peroxidase, the sections and cells were incubated with normal goat serum for $1 \mathrm{~h}$, then incubated with YAP (H-125) (sc-15407; Santa Cruz) (1:50) or p-YAP (Ser127) antibody (\#4911; Cell Signaling Technology) (1:200) at $4^{\circ} \mathrm{C}$ overnight. After washing three times in phosphate-buffered saline (PBS), sections were incubated with a secondary antibody $\left(37^{\circ} \mathrm{C}\right.$ for $1 \mathrm{~h}$ ) (K5007; Dako). DAB horseradish peroxidase color was developed for $20 \mathrm{sec}$ (K5007; Dako).

Real-time PCR. Total RNA was extracted with TRIzol (no. 1382739; Invitrogen, USA). The concentration of RNA was measured by spectrophotometry. The A260/280 ratios of the RNA samples ranged from 1.91 to 2.03. An aliquot (300 ng) of purified RNA from each sample was reverse transcribed to cDNA using a PrimeScript RT reagent kit (code: DRR037A; Takara, Dalian, China), followed by real-time quantitative
PCR (SYBR, code: DRR041A; Takara) in a LightCycle (2.0) system (Roche; software version 3.5). The amplification conditions were: $95^{\circ} \mathrm{C}$ for $30 \mathrm{sec}, 40$ cycles at $95^{\circ} \mathrm{C}$ for $5 \mathrm{sec}$, and $62^{\circ} \mathrm{C}$ for $30 \mathrm{sec}$. Melt curves yielded a single melt peak for all template reactions. Details of the primers are listed in Table I. All primers were designed by the Sangon Company (Shanghai, China) and used according to the manufacturer's instructions. Primer specificity was checked using Primer BLAST (http://www.ncbi.nlm.nih.gov/tools/primer-blast/ index.cgi?LINK_LOC=BlastHome).

For data analysis, the relative quantification was accomplished using a double standard curve method (5-fold serial dilution) and comparison of the $\mathrm{Ct}$ values. The $\mathrm{Ct}$ values of the samples were normalized to an appropriate endogenous housekeeping gene. Each measurement was repeated at least two times.

Western blot analysis. Samples were lysed in RIPA buffer (P0013B; Beyotime, Suzhou, Jiangsu, China) with a protease inhibitor (no. 11873580001; Roche, Penzberg, Germany). Protein concentrations were determined by the BCA method (10) (P0010; Beyotime). At each time point, total protein from pooled liver samples from at least three rats was measured.

For SDS-polyacrylamide gel electrophoresis (10\% gel), $40 \mu \mathrm{g}$ protein was boiled for $10 \mathrm{~min}$ prior to loading. The proteins were transferred onto nitrocellulose membranes (0.45 $\mu \mathrm{m}$; Whatman, Clifton, NJ, USA) by the semi-dry transfer system (Bio-Rad, USA). After blocking, the membrane was probed with YAP (H-125) (1:300), TGF- $\beta 1$ antibody (ab64715; Abcam, UK) $(1.5 \mu \mathrm{g} / \mathrm{ml})$, and $\beta$-actin antibody (Immunocreate, USA) (1:500). Secondary antibodies against rabbit (no. 31460; Thermo Fisher, USA) (1:30,000) and mouse (115-035-003; Jackson, USA) (1:10,000) IgG were used. Signals were amplified by ECL detection reagents (no. 34079; Thermo Fisher). Protein levels were determined semi-quantitatively using Quantity One image software (version 4.4.0).

Statistical analysis. Statistical analysis was performed using the SPSS 13.0 program. Multiple comparisons (Tukey HSD test) and ANOVA were used.

\section{Results}

Quantitation of YAP protein levels in rat regenerating liver. The levels of YAP protein in regenerating rat liver tissues were measured by Western blotting (Fig. 1). At each time point, liver samples from at least three rats were pooled. YAP levels 
A

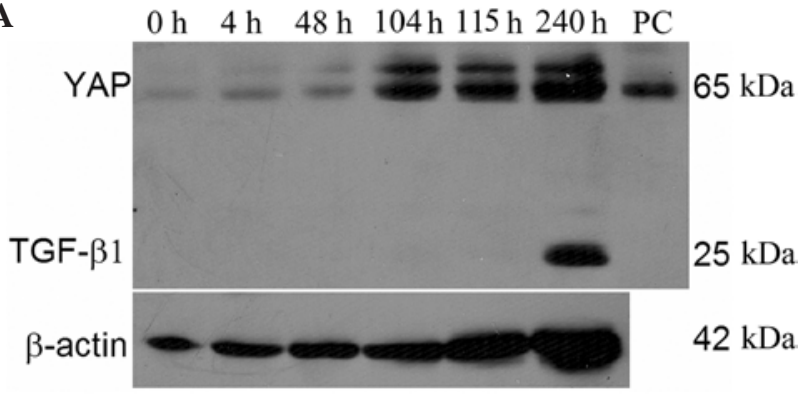

B

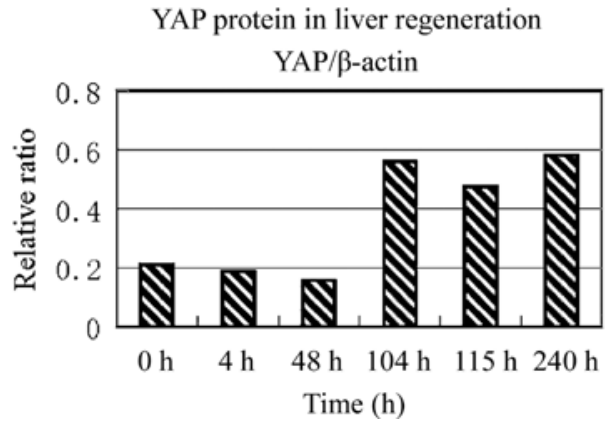

Figure 1. Levels of YAP following PH in rat liver determined by Western blotting. (A) Western blot analysis for YAP and $\beta$-actin was performed using the same sample on different membranes. Positive control (PC) using NIH3T3 in cell lysis solution. (B) Analysis of the peak area of the YAP protein band relative to $\beta$-actin using Quantity One imaging software for the same sample on different membranes.

increased from $104 \mathrm{~h}$ following $\mathrm{PH}$. There was no significant difference among the values at 104, 115 and $240 \mathrm{~h}$. Since the entire process of rat liver regeneration lasts 5 to 7 days (9), YAP protein levels were studied for up to 10 days following $\mathrm{PH}$.
$Y A P$ protein levels increased in rat regenerating liver. The IHC analysis of YAP in the regenerating liver at 0, 4, 48 and $104 \mathrm{~h}$ following $\mathrm{PH}$ are shown in Fig. 2. YAP expression was diffuse at 0,4 and $48 \mathrm{~h}$. Its expression increased significantly at $104 \mathrm{~h}$. These data are consistent with the Western blotting results shown in Fig. 1.

YAP $m R N A$ levels decreased in regenerating liver. Real-time PCR was performed to measure YAP mRNA levels in regenerating liver tissue. As shown in Fig. 3A, relative YAP mRNA (YAP/ $\beta$-actin) levels decreased slightly during the five phases of the regeneration over the course of 10 days. An ANOVA test indicated that the effect of liver regeneration on YAP mRNA was statistically significant $(\mathrm{P}=0.017)$. Multiple comparisons (the Tukey HSD test) at the 5\% level for two-tailed comparisons indicated that there were statistically significant differences between values at the baseline $(0 \mathrm{~h})$ vs. the values at the three time points: 4, 104 and $240 \mathrm{~h}$. However, there were no significant differences between values at the baseline, $48 \mathrm{~h}$ or $115 \mathrm{~h}$, and this result was the same as that observed in Fig. 3B, which was evaluated by $\mathrm{Ct}$ values. YAP mRNA levels in regenerating liver did not increase, but rather decreased, particularly at 4 , 104 and $240 \mathrm{~h}$.

Figs. 3A and B show that YAP mRNA levels at the baseline vs. 48 and $115 \mathrm{~h}$ were not significantly different. TGF- $\beta 1 \mathrm{mRNA}$ was used as a control in Fig. $3 \mathrm{C}$. Ct values (TGF- $\beta 1 \mathrm{mRNA}$ ) revealed a significantly decreasing trend over the three time points. TGF- $\beta 1$ mRNA levels revealed a significant increasing trend from the baseline to $115 \mathrm{~h}$ during liver regeneration in contrast to YAP mRNA levels. These results provide evidence that YAP mRNA levels do not change with increasing TGF- $\beta 1$ mRNA levels during liver regeneration. This is consistent with the results of an earlier study which revealed that following $\mathrm{PH}, 17$ of 19 genes examined by a nuclear run-on assay demonstrated no change in transcriptional activity during the first $96 \mathrm{~h}$ of regeneration (11).

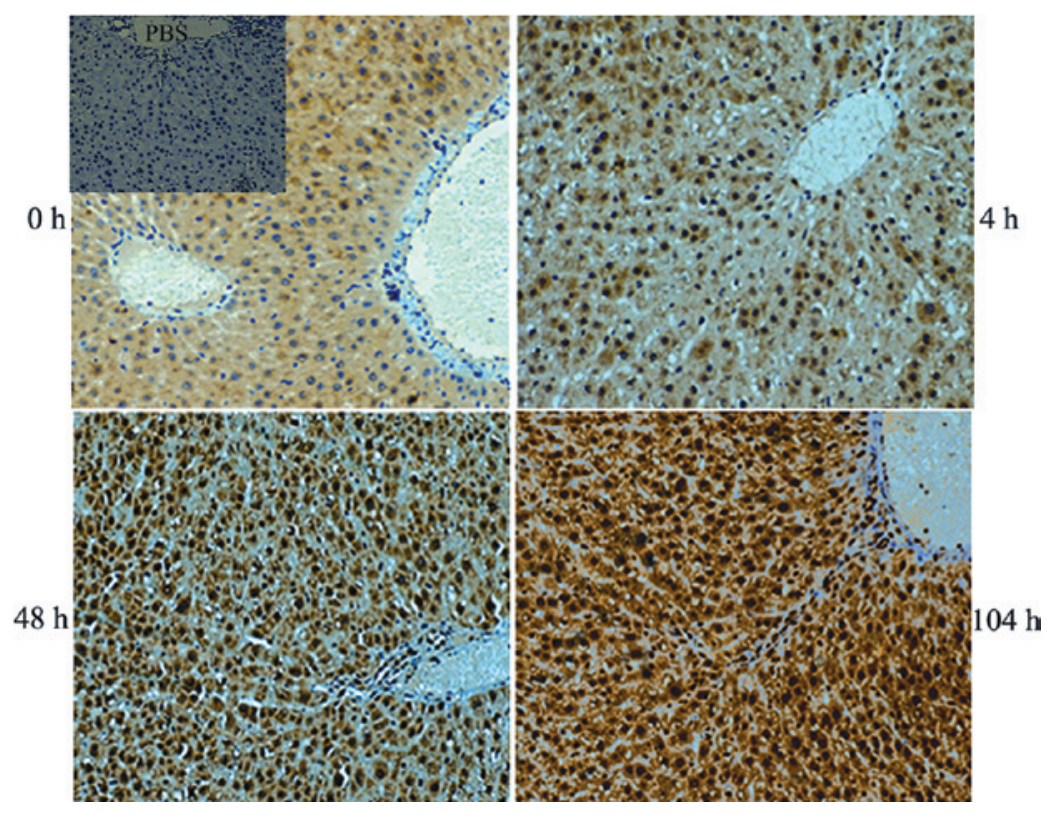

Figure 2. Expression of YAP in rat regenerating liver using immunohistochemistry (x100). Levels of YAP after PH in rat liver at four time points. The top left inset in the $0 \mathrm{~h}$ panel represents the PBS (negative) control. 
A

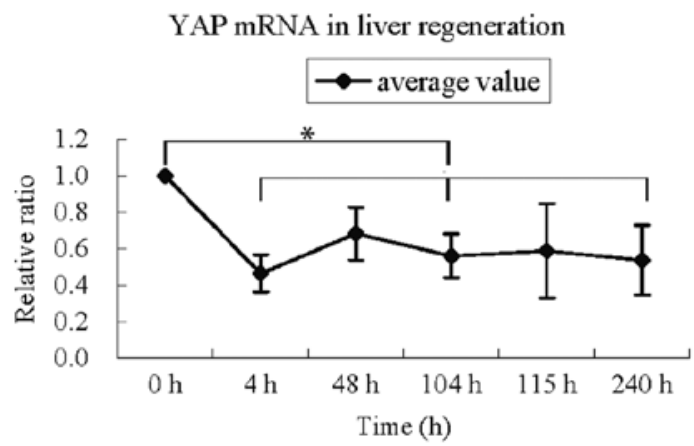

B

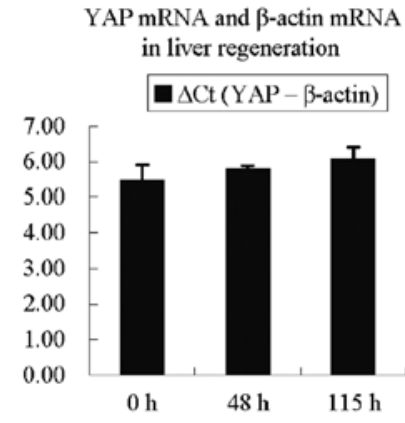

C

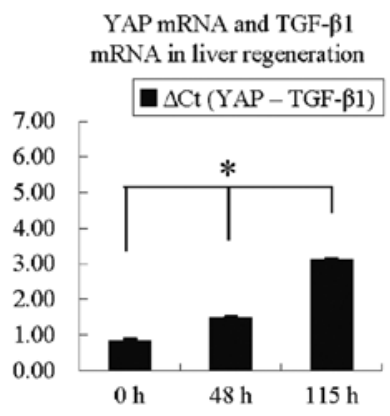

Figure 3. YAP and TGF- $\beta 1$ mRNA levels following PH in rat liver. (A) Real-time PCR data presented as an average of the relative ratio of all samples, including the standard error of the average value. These results are from three experiments with various total RNA samples. "Multiple comparisons (Tukey HSD test), P-values for samples $1-2=0.013 ; 1-4=0.045$; $1-6=0.035$. (B) Real-time PCR values for YAP- $\beta$-actin at three time points $(P>0.05)$. (C) Real-time PCR values for YAP and TGF- $\beta 1$ at the three time points were significantly different $(\mathrm{P}<0.05)$. $\mathrm{B}$ and $\mathrm{C}$, the average values of two experiments.

Overexpression of YAP accompanied by high levels of YAP $m R N A$ in HCC tissue. In HCC, YAP protein levels were higher than those in PCT (Fig. 4A). Furthermore, in PCT, YAP protein levels in the cytoplasm were higher than those in the nuclei. Most of the YAP in PCT was not involved in promoting liver cell proliferation.

We measured YAP mRNA levels in six matched HCC and PCT tissue samples (Fig. 4B). The amount of total RNA and cDNA was the same for each reaction. For all paired samples, the YAP and $\beta$-actin mRNA were amplified. A double-standard curve method was prepared for relative quantification. Levels of YAP mRNA were found to be significantly elevated in HCC tissue samples compared with the PCT counterparts (Fig. 4B).

\section{Discussion}

The present study provides a novel insight into the role of YAP in liver regeneration. The results demonstrated that YAP
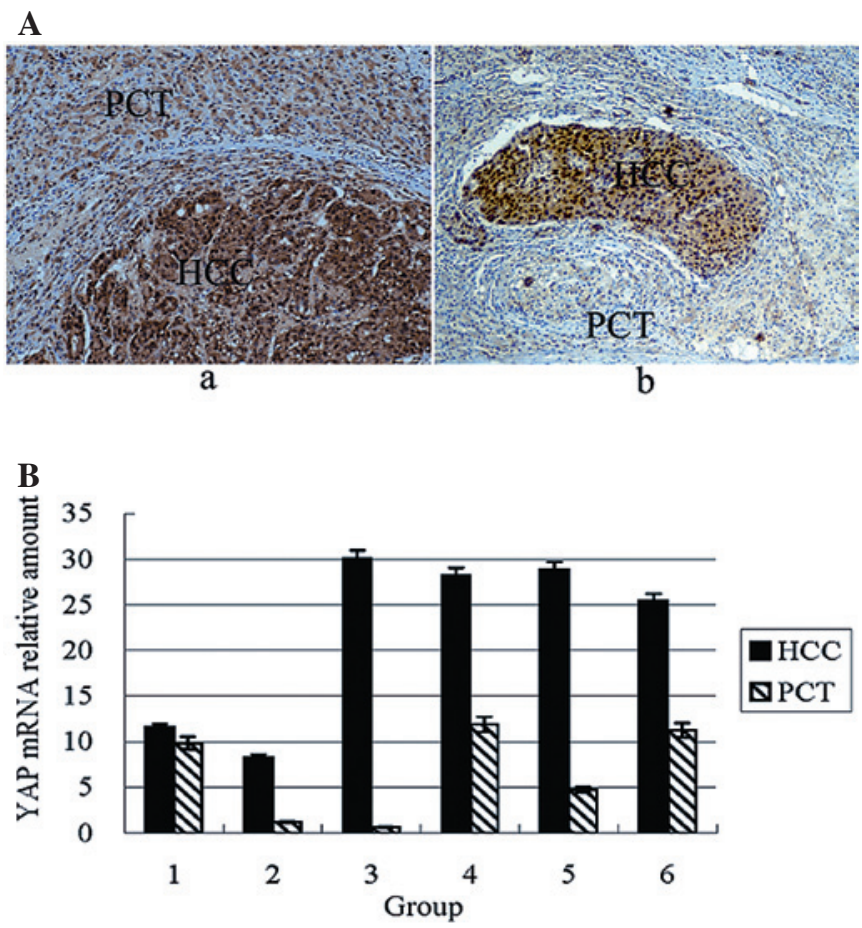

Figure 4. YAP mRNA and protein levels in HCC and PCT. (A) Immunohistochemical staining of anti-YAP antibody in paired (a) HCC and (b) para-cancerous tissue (x100). (B) Real-time PCR assay of YAP in HCC and PCT. $\beta$-actin mRNA levels were used as internal controls. HCC samples of group 2 and 3 in B are the same as in panels $a$ and $b$ in $A$.

protein levels increased in regenerating liver, particularly in the late stages of the study. However, levels of YAP mRNA were not high in this study and decreased slightly over time. On the contrary, control TGF- $\beta 1$ mRNA levels increased in the regenerating liver over time.

Although a previous study (1) showed that YAP gene overexpression led to immediate organomegaly, in the present study, YAP protein levels increased in liver regeneration independently of the high levels of mRNA. This phenomenon indicated that the regulation of YAP in liver regeneration was not at the traditional transcriptional level, possibly due to post-transcriptional or non-transcriptional regulation. Liver cells are known to use various post-transcriptional regulatory mechanisms such as alternative splicing (12), RNA localization (13), RNA stabilization and non-coding RNAs (14) to temporally and coordinately influence the rate of protein synthesis. Results from half-life determinations have indicated that changes in mRNA stability play a critical role in regulating transcript levels during liver regeneration (11). LATS1 (large tumor suppressor 1), a kinase that phosphorylates YAP, promotes YAP protein degradation (15). Therefore, besides post-transcriptional regulation, a decrease in YAP protein degradation may be another alternative explanation for the present data.

Overexpression of YAP could aberrantly activate the target gene CTGF, which is responsible for cell proliferation (16). It is not clear whether there are specific molecules that trigger or suppress liver regeneration to maintain proper liver size. One study noted that the loss of a YAP suppressor may lead to uncontrolled cell growth (1). Those results and the present study suggest that YAP participates in controlling liver size. 
Further study will be required to discover new mechanisms of the YAP gene post-transcriptional regulation.

A previous study revealed that YAP mRNA levels were significantly elevated (4-fold) in the majority of HCC tumor tissues compared with adjacent non-tumor tissues (2). In the present study, YAP expression demonstrated an increasing trend from PCT to HCC tissues. More significantly, YAP was concentrated in the nuclei of liver cancer cells. This indicates that the nucleoplasm transport of YAP is a significant link in tumor progression in HCC. This phenomenon also occurred in the course of liver regeneration. Therefore, the similarity suggests that cancer cells in regenerating liver may possess a powerful nucleoplasm transport mechanism from the cytoplasm to the nucleus.

A recent study provided clinical evidence that YAP is an independent prognostic marker for overall survival in HCC patients (2). The expression of YAP was strongly correlated with tumor recurrence. These reports are consistent with our hypothesis that YAP protein has a significant proliferative effect through a liver regeneration mechanism on residual tumors.

Clinical evidence suggests that stimulation of tumor growth may occur following resection of liver tumors (17), and that liver regeneration could accelerate cancer cell proliferation (18). The growth rate of metastases exceeded that of the normal liver tissues when patients sustained right portal embolization (19). We suggest that liver cancers expressing high levels of YAP will be sensitive to liver regeneration as YAP-positive HCC would generate higher levels of YAP following $\mathrm{PH}$.

In conclusion, liver regeneration elevates YAP protein levels without elevating levels of YAP mRNA. In HCC, the overexpression of YAP is usually accompanied by a high level of YAP mRNA. Therefore, when liver regeneration occurs in the presence of YAP-positive HCC, most of the cancer cells are stimulated by regeneration factors. The double effect of the high levels of YAP mRNA and the high intensity of non-transcriptional regulation could account for the elevated YAP protein levels. The increase in YAP protein in the nuclei may be involved in the early stages of recurrence of HCC.

\section{Acknowledgements}

We are grateful to Qingliang Wang, Ling Shuai, Peng Jiang, Yujun Zhang, Yan Jiang, and all members from the Hepatobiliary Surgery Laboratory of Southwest Hospital that provided helpful input. This study was supported by a grant from the National Science and Technology Major Project of China (2008ZX10002-026).

\section{References}

1. Dong JX, Feldmann G, Huang JB, et al: Elucidation of a universal size-control mechanism in Drosophila and mammals. Cell 130: 1120-1133, 2007

2. Xu MZ, Yao TJ, Lee NYP, et al: Yes-associated protein is an independent prognostic marker in hepatocellular carcinoma. Cancer 115: 4576-4585, 2009.

3. Tordjmann T: Hippo signalling: liver size regulation and beyond. Clin Res Hepatol Gastroenterol 35: 344-346, 2011.

4. Zhao B, Wei X, Li W, et al: Inactivation of YAP oncoprotein by the Hippo pathway is involved in cell contact inhibition and tissue growth control. Genes Dev 21: 2747-2761, 2007.

5. Lee KP, Lee JH, Kim TS, et al: The Hippo-Salvador pathway restrains hepatic oval cell proliferation, liver size, and liver tumorigenesis. Proc Natl Acad Sci USA 107: 8248-8253, 2010.

6. Zender L, Spector MS, Xue W, et al: Identification and validation of oncogenes in liver cancer using an integrative oncogenomic approach. Cell 125: 1253-1267, 2006.

7. Lencioni RA, Allgaier HP, Cioni D, et al: Small hepatocellular carcinoma in cirrhosis: randomized comparison of radio-frequency thermal ablation versus percutaneous ethanol injection. Radiology 228: 235-240, 2003.

8. Meredith K, Haemmerich D, Qi C and Mahvi D: Hepatic resection but not radiofrequency ablation results in tumor growth and increased growth factor expression. Ann Surg 245: 771-776, 2007.

9. Higgins GM: Experimental pathology of the liver: restoration of the liver in the white rat following partial surgical removal. Arch Pathol 12: 186-202, 1931.

10. Liu XS, Jiang J, Jiao XY, Wu YE, Lin JH and Cai YM: Lycorine induces apoptosis and down-regulation of Mcl-1 in human leukemia cells. Cancer Lett 274: 16-24, 2009.

11. Kren BT, Trembley JH and Steer CJ: Alterations in mRNA stability during rat liver regeneration. Am J Physiol 270: 763-777, 1996.

12. Zweifel M, Breu K, Matozan K, Renner E, Welle M, Schaffner T and Clavien PA: Restoration of hepatic mast cells and expression of a different mast cell protease phenotype in regenerating rat liver after 70\%-hepatectomy. Immunol Cell Biol 83: 587-595, 2005.

13. Komuves LG, Feren A, Jones AL and Fodor E: Expression of epidermal growth factor and its receptor in cirrhotic liver disease. J Histochem Cytochem 48: 821-830, 2000.

14. Matouk IJ, DeGroot N, Mezan S, Ayesh S, Abu-lail R, Hochberg A and Galun E: The H19 non-coding RNA is essential for human tumor growth. PloS One 2: e845, 2007.

15. Zhao B, Li L, Tumaneng K, Wang CY and Guan KL: A coordinated phosphorylation by Lats and CK1 regulates YAP stability through SCF (beta-TRCP). Genes Dev 24: 72-85, 2010.

16. Zhao B, Ye X, Yu J, et al: TEAD mediates YAP-dependent gene induction and growth control. Genes Dev 22: 1962-1971, 2008.

17. Schindel DT and Grosfeld JL: Hepatic resection enhances growth of residual intrahepatic and subcutaneous hepatoma, which is inhibited by octreotide. J Pediatr Surg 32: 995-998, 1997.

18. Barbeito CG, Garcia MN, Flamini MA, Andrini LB and Badran AF: Effect of partial and sham hepatectomy on the growth of hepatocellular carcinoma. J Exp Clin Cancer Res 20: 153-158, 2001.

19. Seymour K, Manas D and Charnley RM: During liver regeneration following right portal vein embolization the growth rate of liver metastases is more rapid than that of the liver parenchyma. Br J Surg 86: 1482-1483, 1999. 